\title{
ARTICLE
}

\section{Direct dose measurement of patients during pediatric computed tomography examination}

\author{
Hiroaki Hayashi ${ }^{\mathrm{a}, \mathrm{f}^{*}}$, Emi Tomita ${ }^{\mathrm{b}, \mathrm{f}}$, Sota Goto ${ }^{\mathrm{b}, \mathrm{f}}$, Natsumi Kimoto,f, Keiji Tadac ${ }^{\mathrm{c}}$, Ryosuke Kasai ${ }^{\mathrm{d}}$, Yuki Kanazawa ${ }^{\mathrm{a}}$, \\ Yoshiki Mihara $^{\mathrm{a}}$, Takashi Asahara ${ }^{\mathrm{a}}$, Tohru Okazaki ${ }^{\mathrm{e}}$, Takuya Hashizume ${ }^{\mathrm{e}}$ and Vergil Lorenzo E. Cruz \\ ${ }^{a}$ Graduate School of Health Sciences, Tokushima University, 3-18-15 Kuramoto, Tokushima, Japan; ${ }^{b}$ School of Health Sciences, \\ Tokushima University, 3-18-15 Kuramoto, Tokushima, Japan; ' Shimane University Faculty of Medicine, 89-1 Enya, Izumo, Shimane, \\ Japan; ${ }^{d}$ Tokushima University Hospital, 3-18-15 Kuramoto, Tokushima, Japan; ${ }^{e}$ Nagase Landauer, Ltd., Block C22-1, Suwa,

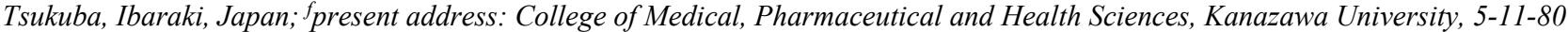 \\ Kodatsuno, Kanazawa, Japan
}

Even though various dose reduction techniques are applied on current computed tomography (CT) examination, patient dose is not measured. In this study, we measured the patient dose (surface dose) of neonates and infants and focused on using an optically stimulated luminescence (OSL) dosimeter to carry out the measurement since this dosimeter does not interfere with the medical image. For each CT examination, we applied a volumetric scanning technique in which we only needed one rotational scanning for obtaining an image within the length of $16 \mathrm{~cm}$ with 320 slices. First, using a neonate phantom, we examined the effect of placing patients at different positions on the measured doses. Second, we measured the patient dose using OSL dosimeters with thirteen dosimeters placed on each patient. During clinical CT examinations, exposure doses were automatically determined using an automatic exposure control system. In order to derive the differences of doses between patients and phantom, we examined the trend of values in which exposure doses were divided by the computed tomography dose index. We found that patient data showed a different trend when compared with the data obtained from the neonate phantom experiment. This result shows that this basic knowledge will be valuable in deciding proper irradiation conditions.

Keywords: CT; OSL dosimeter; direct dose measurement; volumetric scan; pediatric examination

\section{Introduction}

Pediatric computed tomography (CT) examination is widely performed in hospitals and clinics. During CT examinations, reducing the exposure dose is important from the radiation safety point of view; especially for radiosensitive neonates and infants, some researchers are alarmed about the increasing radiation doses [1-3]. An area detector CT [4] is currently being used for these examinations, because the scan mode archives high reduction rate of exposure doses with short scanning time $[5,6]$. There are many reports concerning Toshiba's 320 -detector row CT [5,6], in which a scan length of 160 $\mathrm{mm}$ can be applied for volumetric scanning.

In addition, the current $\mathrm{CT}$ examinations are operated under automatic exposure control (AEC) system [7,8], in which exposure doses (tube currents) are continuously varied during scanning. The exposure doses were determined automatically so as to obtain a proper CT image. As a result, exposure doses varied for each patient. We consider that patient dose should be properly

*Corresponding author. Email: hayashi.hiroaki@staff.kanazawa-u.ac.jp measured in clinical situations. An optically stimulated luminescence (OSL) dosimeter $[9,10]$ is one of the proper detectors for actual dose measurements.

In this study, our focus was on cardiac imaging in neonates and infants using the volumetric 320-detector row $\mathrm{CT}$. We then examined the applicability of the OSL dosimeter to the clinical CT examinations using a neonate phantom, and are the first to report clinical data results.

\section{Materials and methods}

\subsection{Phantom study}

We used a 320-detector row CT (Aquilion ONE ${ }^{\mathrm{TM}}$, Toshiba Medical Systems, Otawara, Japan) and a neonate phantom (PH-50, Kyoto Kagaku Co. Ltd., Kyoto, Japan). We simulated a cardiac examination of neonates and infants, in which volumetric scan mode was applied [5-6]; the scanning range was $160 \mathrm{~mm}$, and the upper limit was set at the chin. The rotational speed of the X-ray tube, tube voltage, and tube current were $0.275 \mathrm{~s}$ per rotation, $80 \mathrm{kV}$, and $100 \mathrm{~mA}$, respectively. 
In order to measure surface doses, OSL dosimeters (nanoDot, Nagase Landauer, Ltd., Ibaraki, Japan) [9,10] were placed on the skin. 6 dosimeters were used for the chest region, 4 dosimeters were placed around the eye lens, 4 dosimeters were used for wrists and ankles, and 1 dosimeter was placed in the genital area. The exposed OSL dosimeters were analyzed with a portable-type OSL reader (microStar, Nagase Landauer, Ltd., Ibaraki, Japan).

Using the phantom, we examined the impact of setting at the abnormal positions (off-centered: $+/-1 \mathrm{~cm}$ and rotating: $+/-10$ degree) on doses, because during clinical examinations there is a possibility that positioning becomes abnormal. In order to evaluate the repeatability of this experiment, it was carried out five times.

\subsection{Clinical study}

We examined 6 cases for the clinical study; ages in month were 2-23 $(13.8+/-9.5)$, and weights $(\mathrm{kg})$ were 3.9-11.3 (8.2+/-2.8). All patients have congenital heart problems, and the aim of this CT examination was to keep track of the position and shape of the heart, pulmonary artery, pulmonary vein, vital artery, and coronary artery. The same CT scanner used for the phantom study was also used during the clinical examination; scanning parameters were almost identical, however tube current for each examination was determined by AEC.

The 13 OSL dosimeters were placed in a similar way as described above; only two dosimeters are placed around eye lids, and for the other positions, the same number of dosimeters were used. This study was permitted by Institutional Review Board of our hospital.

\section{Results and discussion}

\subsection{Phantom study}

Figure 1 shows the results of the phantom study. As shown in Figure 1 (a), dose distribution of the normal position was presented; the left picture shows a photograph from a top view, and measured doses are indicated in the right in which a scanning region is displayed by the gray-scaled X-ray image (coronal axis image). We can identify that the 6 dosimeters placed in the scanning region show relatively high values. On the contrary, the other dosimeters outside the scanning region show relatively low values. Figures 1 (b), (c), (d) and (e) show similar results for the off-centered positions $(+/-1 \mathrm{~cm})$, and rotated positions ( $+/-10$ degree).

In order to evaluate these trends quantitatively, the measured values for 5 individual experiments and an averaged value of these experiments are shown in Figure 2. The error bar of the averaged dose shows the standard deviations for 5 runs. It is clear that there are no significant differences between observed doses in Figures 2 (a)-(c). From this fact, we evaluated that impact of different positions on the measured doses during the pediatric $\mathrm{CT}$ examination seems to be small.
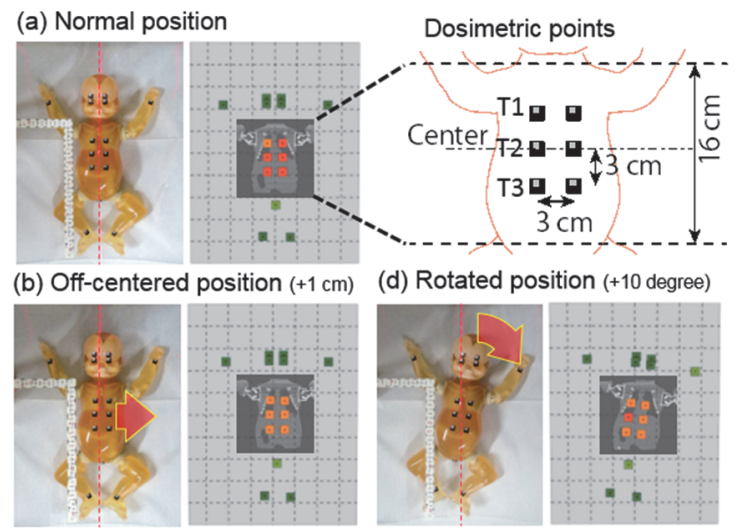

(c) Off-centered position $(-1 \mathrm{~cm})$

(e) Rotated position (-10 degree)
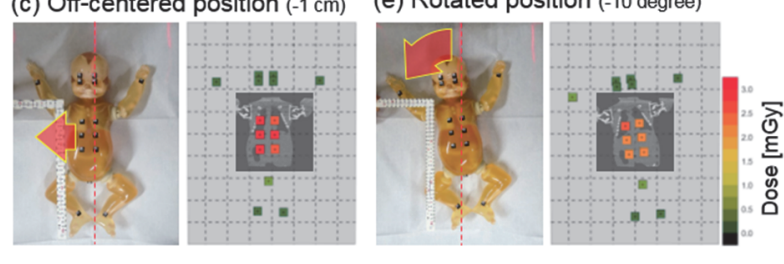

Figure 1. Result of a phantom study. The upper Figure (a) shows original (standard) position and dose distribution. Figures of (b)-(e) show abnormal positions.

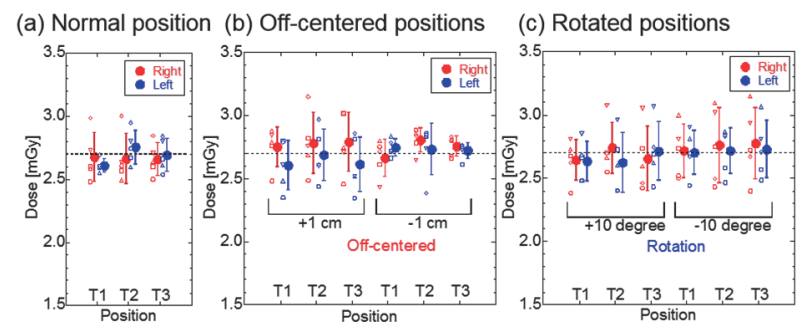

Figure 2. Comparison of observed doses for three different positions: (a) normal position, (b) off-centered positions, and (c) rotated positions. There are no significant differences in observed data. Results of the 5 individual experiments are presented by open symbols and averaged values are plotted as closed symbols.

\subsection{Clinical study}

Figure 3 shows a typical result from the clinical study: an 8 months' patient with $7.5 \mathrm{~kg}$. The observed doses in the irradiation area (thoracic region) are 4.3-4.8 mGy caused mainly by direct X-rays. On the other hand, exposure doses caused by scattering X-rays were less than a few percent when compared with the doses inside the irradiation area: eye lens $0.15 \mathrm{mGy}$, gonad $0.11 \mathrm{mGy}$, wrists $0.13-0.16 \mathrm{mGy}$ and ankles $0.02-0.03 \mathrm{mGy}$. The CT images on the right demonstrates an axial cross section in which the OSL dosimeters were placed on the skin; we designated them as $\mathrm{T} 1, \mathrm{~T} 2$ and $\mathrm{T} 3$ for upper, middle and lower positions, respectively. It is clear that image density concerning the area of the OSL dosimeter (CT value) is similar with those of soft-tissue material, which have an effective atomic number of approximately 7.0 [11]. This means that the OSL dosimeter has a relatively small effective atomic number 
and that is why the dosimeter does not interfere with the CT image. This figure shows evidence that the OSL dosimeter can be applied to measure doses during clinical CT examination in that they are compatible with obtaining medical images.

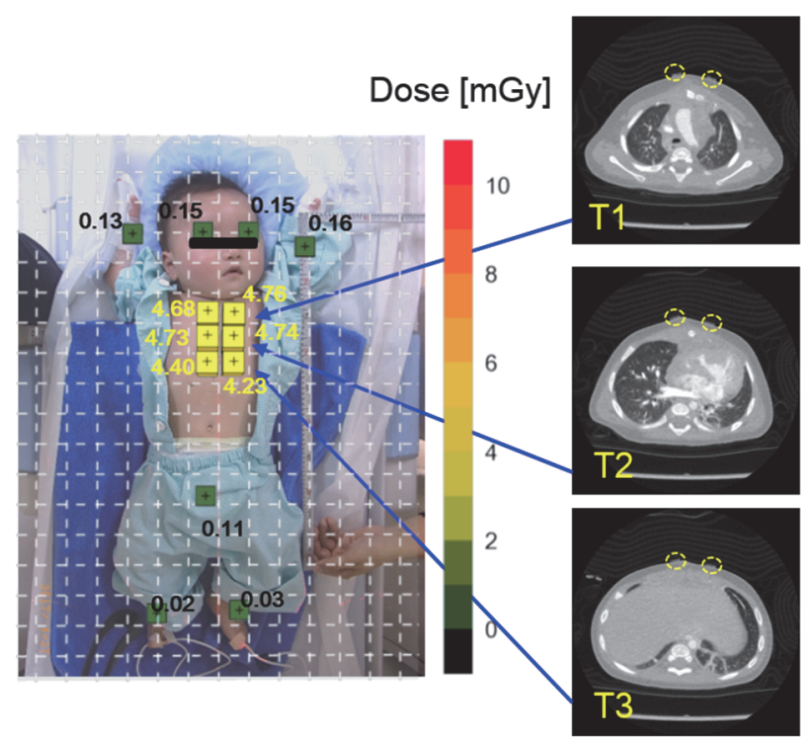

Figure 3. Typical result of dose measurement for clinical pediatric CT examination. In the left figure, measured doses are overlapped with the photograph, and the right figures show $\mathrm{CT}$ images. OSL dosimeters were placed in the regions, which are identified by broken circles.

Figure 4 shows a comparison between patient weights and measured doses at $\mathrm{T} 1, \mathrm{~T} 2$ and $\mathrm{T} 3$ positions. All clinical data concerning the 2-23 month patients were included, as well as the neonate phantom data. Note that the phantom data in this figure was performed under clinical examinations condition using AEC. The plot shows rough relationships between $\mathrm{X}$ - and Y-axes; the observed doses proportionally increased as weights increased. In these plots, data have an uncertainty of approximately $15 \%$, because systematic uncertainty was mainly affected and statistical uncertainty is negligibly small. In a previous study [9], we estimated that the systematic uncertainty of the OSL dosimeter was at most $15 \%$ when the dosimeter was used under the condition in which energy and scattering angles were not uniformly determined. The present results deviated evenly from the fitted line, and this indicates that error estimations work well.

In Figure 4, the reasons for differences between the neonate phantom and clinical patient data were unclear. This is because for these data, exposure doses were determined by AEC. The AEC installed in the Toshiba's CT scanner $[7,8]$ practically determined exposure doses by a preliminary scanned image, which is called "scanogram" and this image is generally used to determine scanning regions. It is unclear whether the same patient parameters will be used to apply the same exposure doses, and these uncertainties are included in the data presented in Figure 4.

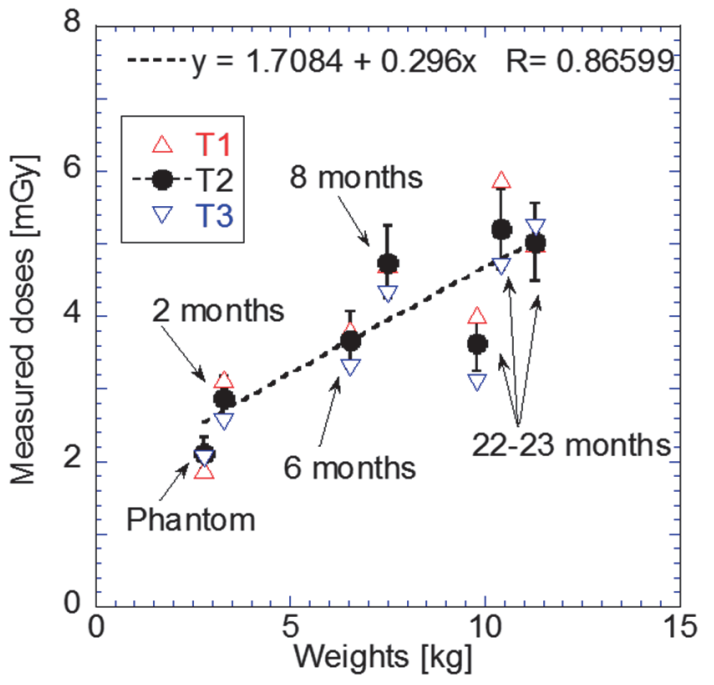

Figure 4. Relationship between measured doses and weight of patients. Notations of T1, T2, and T3 are defined in Figure 3.

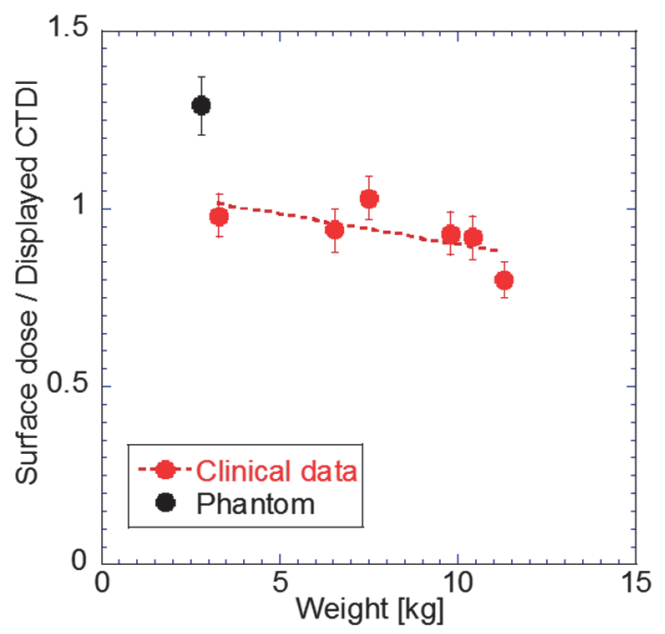

Figure 5. Trend of the ratios, in which measured surface doses were divided by displayed CTDI values. There are significant differences between phantom and clinical patient data.

After the examination was completed, the CT scanner reported a computed tomography dose index (CTDI) value [12]. The CTDI value is known as a reference value of exposure dose, which is determined by the manufacturer using the special CTDI phantom. In order to derive the differences between phantom and patient data taking into consideration variations of exposure doses for each patient, we calculated the ratios of the surface doses divided by the CTDI values. The relationship between the ratios and weights are represented in Figure 5. In this graph, red and black circles show clinical and phantom data, respectively. The dashed red line shows a linear function which fits to the clinical data; they show good correlation. It is an interesting point that the linear function shows a negative gradient; namely, the ratios of surface dose divided by CTDI values show a larger value for lighter patients and for heavier patients the ratios have smaller values. We think the analysis of the trends gives us basic 
knowledge of exposure. A detailed analysis is now in progress. Other important information in Figure 5 is that the phantom data shows completely different trends when compared with clinical patient data, nevertheless the neonate phantom was made based on $\mathrm{CT}$ data to mimic a realistic patient.

In general, exposure doses were estimated from the experiment using a CTDI phantom, whose shape is cylindrical, and scientists consider that the CTDI value is just an index value [12]. Recently many manufactures fabricate realistic phantoms instead of using patients, and it is expected to estimate exposure doses when using them. These studies are valuable for management of exposure dose during a pediatric CT examination, but the present study indicates a possibility of discrepancies between phantoms and patients.

\section{Conclusion}

In this study, we measured exposure doses using the OSL dosimeters during CT examinations. From the phantom study, we verified that our procedure works well even when patients were placed at abnormal positions (shifting and rotating positions). Moreover, we measured patient data in a clinic, and we found that there are obvious differences between patient and phantom data.

\section{Acknowledgements}

This work was partially supported by JSPS KAKENHI Grant Number 15K19205. Authors would like to thank to Dr. Ikuo Kobayashi (Nagase Landauer, Ltd.) for valuable discussions concerning the OSL dosimeter, and also thank to Ms. Saki Kageyama and Ms. Haruna Oka (Shimane University Hospital) for their contribution of performing the clinical examinations.

\section{References}

[1] D.J. Brenner, C.D. Elloston, E.J. Hall and W.E. Berdon, Estimated risks of radiation-induced fatal cancer from pediatric CT, American Journal of Radiology 176 (2001), pp. 289-296. DOI: 10.2214/ajr.176.2.1760289

[2] D.J. Brenner, Estimating cancer risks from pediatric CT: going from the qualitative to the quantitative. Pediatric Radiology 32 (2002), pp. 228-231 DOI: $10.1007 / \mathrm{s} 00247-002-0671-1$

[3] L.F. Donnelly, K.H. Emery, A.S. Brody, T. Labor, V.M. Gylys-Morin, C.G. Anton, S.R. Thomas and D.P. Frush, Minimizing radiation dose for pediatric body applications for single-detector helical CT: Strategies at a Large Children's Hospital, American Journal of Radiology 176 (2001), pp.303-306 DOI: 10.2214/ajr.176.2.1760303

[4] M. Mahesh, Advances in CT technology and application to pediatric imaging, Pediatric Radiology 41 (2011), pp. S493-S497. DOI: 10.1007/s00247-011-2169-1

[5] E. Sorantin, M. Riccabona, G. Stücklschweiger, H. Guss and R. Fotter, Experience with volumetric (320 rows) pediatric CT, European Journal of Radiology 82 (2013), pp. 1091-1097. DOI: 10.1016/j.ejrad.2011.12.001

[6] L.J.M. Kroft, J.J.H. Roelofs and J. Geleijns, Scan time and patient dose for thoracic imaging in neonates and small children using axial volumetric 320-detector row compared to helical 64-, 32-, and 16- detector row CT acquisitions, Pediatric Radiology 40 (2010), pp. 294-300. DOI: 10.1007/s00247-009-1436-x

[7] T. Yamashiro, T. Miyar, O. Honda, H. Kamiya, K. Murata, Y. Ohno, N. Tomiyama, H. Moriya, M. Koyama, S. Noma, A. Kamiya, Y. Tanaka and S. Murayama, Adaptive iterative dose reduction using three dimensional processing (AIDER3D) improves chest CT image quality and reduces radiation exposure, PLOS one 9 (2014), pp. e105735(1-10). DOI:10.1371/journal.pone.0105735

[8] Y. Yamada, M. Jinzaki, T. Hosokawa, Y. Tanami, H. Sugiura, T. Abe and S. Kuribayashi, Dose reduction in chest $\mathrm{CT}$ : Comparison of the adaptive iterative dose reduction $3 \mathrm{D}$, adaptive iterative dose reduction and filtered back projection reconstruction techniques, European Journal of Radiology 81 (2012), pp. 4185-4195. DOI: 10.1016/j.ejrad.2012.07.013

[9] K. Takegami, H. Hayashi, H. Okino, N. Kimoto, I. Maehata, Y. Kanazawa, T. Okazaki and I. Kobayashi, Practical calibration curve of small-type optically stimulated luminescence (OSL) dosimeter for evaluation of entrance skin dose in the diagnostic X-ray region, Radiological Physics and Technology 8 (2015), pp.286-294. DOI: $10.1007 / \mathrm{s} 12194-015-0318-1$

[10]K. Takegami, H. Hayashi, K. Yamada, Y. Mihara, N. Kimoto, Y. Kanazawa, K. Higashino, K. Yamashita, F. Hayashi, T. Okazaki, T. Hashizume and I. Kobayashi, Entrance surface dose measurements using a small OSL dosimeter with a computed tomography scanner having 320 rows of detectors. Radiological Physics and Technology. 10 (2017), pp. 49-59. DOI: 10.1007/s12194-016-0366-1

[11]N. Kimoto, H. Hayashi, T. Asahara, Y. Mihara, Y. Kanazawa, T. Yamakawa, S. Yamamoto, M. Yamasaki and M. Okada, Precise material identification method based on a photon counting technique with correction of the beam hardening effect in X-ray spectra, Applied Radiation and Isotopes 124 (127), pp. 16-26. DOI: 10.1016/j.apradiso.2017.01.049

[12]C.H. McCollough, S. Leng, L. Yu, D.D. Cody, J.M. Boone and M.F. McNitt-Gray, CT dose index and patient dose: They are not the same thing. Radiology 259 (2011), pp. 311-316. DOI: 10.1148/radiol.11101800 